\title{
Reminiscencia y Variables Adaptativas en Personas Mayores
}

\author{
Reminiscence and Adaptive Variables in Older People
}

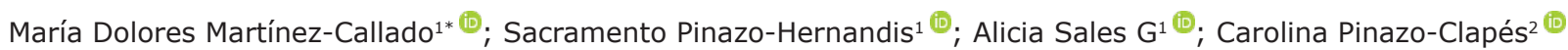

Recibido: 08-11-2020; Aceptado: 22-12-2020; Publicado: 28-12-2020

\section{RESUMEN}

Objetivo. El propósito de esta revisión es conocer y resumir la evidencia sobre la efectividad de la Terapia de Reminiscencia en diferentes variables adaptativas, publicada en revistas científicas en los últimos 5 años. Método. La búsqueda de información relevante se realizó a partir de las bases de datos Web of Science, Current Contents Connect, Derwent Innovations Index, KCI-Korean Journal Database, Medline y Russina Science Index, y Google Scholar. Tras una primera búsqueda de 1689 estudios, se revisan finalmente 13 estudios que seguían los criterios de diseño marcados por los investigadores. Resultados. Los resultados nos muestran que la mayor parte de las investigaciones se realizan en comunidad. Las variables más contempladas son la calidad de vida y el bienestar psicológico. Además, la Terapia de Reminiscencia conecta las experiencias pasadas de las personas mayores, permitiéndoles relatar y compartir sus historias de vida, obtener apoyo emocional y aliviar las emociones negativas, la depresión y la soledad. Conclusiones. Las funciones de la Reminiscencia son relevantes en la vejez. Este trabajo podría ser relevante para motivar la investigación y la intervención sobre el tema de la Reminiscencia y sus beneficios potenciales al mejorar la ansiedad, síntomas depresivos, habilidades cognitivas, bienestar psicológico, satisfacción vital, calidad de vida, compromiso social, capacidad adaptativa, resiliencia, y significado de vida, favoreciendo la integridad y obteniéndose, además, beneficios positivos para la salud en personas mayores.

Palabras clave: Reminiscencia; envejecimiento; vejez; identidad; revisión sistemática; mayores; comunidad; depresión; ansiedad; bienestar; calidad de vida; adaptación.

\section{ABSTRACT}

Objective. The purpose of this review is to know and summarize the evidence on the effectiveness of reminiscence therapy in different adaptive variables, published in scientific journals in the last 5 years. Method. The search for relevant information was carried out using the Web of Science, Current Contents Connect, Derwent Innovations Index, KCI-Korean Journal Database, Medline and Russina Science Index, and Google Scholar databases. After a first search of 1689 studies, 13 studies that followed the design criteria set by the researchers were finally reviewed. Results. The results show us that most of the research is carried out in the community. The most widely considered variables are quality of life and psychological well-being. In addition,

${ }^{1}$ University of Valencia, Faculty of Psychology, Valencia, Spain. domca24@hotmail.com; sacramento.pinazo@uv.es; Alicia.sales@uv.es 2European University, Faculty of Psychology, Valencia, Spain. carolina.pinazo@universidadeuropea.es 
reminiscence therapy connects older people's past experiences, allowing them to relate and share their life stories, gain emotional support, and alleviate negative emotions, depression, and loneliness. Conclusions. The functions of reminiscence are relevant in old age. This work could be relevant to motivate research and intervention on the topic of reminiscence and its potential benefits in improving anxiety, depressive symptoms, cognitive skills, psychological well-being, life satisfaction, quality of life, social commitment, adaptive capacity, resilience, and meaning of life, favoring integrity, and obtaining positive health benefits in older people.

Keywords: Reminiscence; aging; old age; identity; systematic review; greater; community; depression; anxiety; wellness; quality of life; adaptation.

\section{INTRODUCCIÓN}

Durante la vejez, las personas dedican tiempo al recuerdo de acontecimientos pasados y a la elaboración de una narrativa de su historia vital. Erikson (1982) contempla la vejez como una etapa en la que las personas se enfrentan a la tarea de reflexionar y aceptar todas aquellas experiencias del pasado tanto positivas como negativas sobre la vida y la integración de toda la experiencia en un todo con sentido. A esta tarea evolutiva la denominó Integridad. Lograr la integridad significa alcanzar la aceptación del pasado, dotar de significado a la vida, adquirir una identidad yoíca y enfrentarse al futuro con serenidad.

La intervención terapéutica en Reminiscencia ayuda a las personas a la reconciliación con el pasado y al desarrollo de la integridad en la última etapa del ciclo vital. Uno de los autores más importantes en relación con la Terapia de Reminiscencia es Webster (1997) quien la definió como "el recuerdo e interpretación presente de acontecimientos vitales que experimentamos en algún momento de nuestro pasado, generalmente de nuestro pasado lejano".

La Reminiscencia es una actividad psíquica universal necesaria en el envejecimiento y en la vejez, permite recordar pensando o relatando hechos, actos o vivencias del pasado, favorece la integración del pasado con el presente, brinda continuidad, refuerza la identidad, aumenta la autoestima y permite la resignificación (Westerhof, Bohlmeijer y Webster, 2010).

Cortés (2016) la define como el gesto o huella que nos remite a situaciones de nuestro pasado a través de objetos y sensaciones, que permite congelar un instante y salvaguardar los recuerdos de la fugacidad de la memoria.

La Reminiscencia es una herramienta potencialmente eficiente en diferentes variables emocionales: disminuye la ansiedad, mejora síntomas depresivos (Lopes, Afonso y Ribeiro 2016; Van Bogaert et ál., 2016; Duru y Kapucu, 2016) y habilidades cognitivas (Irazoki, García, Sánchez y Franco, 2017; Lök, Bademli y Selçuk-Tosun, 2019).

Son pocas las investigaciones que han estudiado las relaciones entre variables adaptativas e integridad del yo: resiliencia, capacidad adaptativa (Meléndez, Fortuna, Sales y Mayordomo, 2015a), bienestar psicológico, satisfacción vital, calidad de vida, compromiso social (González, Mayordomo, Torres, Sales y Meléndez, 2015) y significado de vida (Bohlmeijer, Westerhof y Emmerik-de Jong, 2008).

Debido a los constantes cambios que se producen durante la etapa de la vejez, es importante dotar de recursos y estrategias a las personas mayores para enfrentarse a estos cambios y desafíos: pérdida de trabajo por jubilación, pérdida de iguales (cónyuges, hermanos, amigos), pérdida de poder adquisitivo y pérdida de salud constituyen una importante fuente de estrés. Cuando las pérdidas representan una serie de fracasos, y la persona cree que no tiene recursos 
suficientes para afrontar la situación, pueden aparecer sentimientos de desesperanza, siendo la Terapia de Reminiscencia una herramienta eficaz para mejorar la capacidad adaptativa.

El propósito de esta revisión es conocer y resumir la evidencia sobre la efectividad de la Terapia de Reminiscencia en diferentes variables adaptativas, publicada en revistas científicas en los últimos 5 años.

\section{MÉTODO}

Selección de los Estudios. La búsqueda de información relevante se realizó a partir de las bases de datos Web of Science, Current Contents Connect, Derwent Innovations Index, KCI-Korean Journal Database, Medline y Russina Science Index y Google Scholar. Las palabras clave utilizadas para consultar fueron "reminiscence" AND/OR "intervention". Tras una primera búsqueda de 1689 estudios, se revisaron finalmente 13 estudios que seguían los criterios de diseño marcados por los investigadores. La búsqueda se realizó en los últimos 5 años (2015-2020), inglés, 1 campo predeterminado, artículos y revistas científicas, cuyo objetivo fue el estudio de variables adaptativas en programas de intervención en Reminiscencia para personas mayores.

Criterios de Inclusión. En la investigación se incluyeron estudios cuyos participantes fuesen personas mayores de 60 años, que conservaran las funciones cognitivas o con deterioro cognitivo leve; trabajos que valoraran variables adaptativas (calidad de vida, satisfacción con la vida, sentido de vida, autoestima, propósito de vida, bienestar psicológico, integridad, afrontamiento y adaptación); ensayos aleatorios o cuasi experimentales con medidas pre y post en las intervenciones grupales, mostrando los efectos de la Reminiscencia a través de resultados estadísticos que confirmaran tales efectos; artículos publicados en revistas de impacto JCR o, al menos, que hubiesen pasado por un proceso de revisión por pares, artículos de acceso abierto, trabajos publicados en inglés, en revistas de diferentes disciplinas (incluyendo Psicología, Enfermería, Medicina, Ciencias Sociales y Educación).

Criterios de Exclusión. Se excluyeron los artículos publicados previos al 2015; estudios cuyo objetivo no era el estudio de variables adaptativas en programas de intervención en Reminiscencia para personas mayores, y artículos cuyos participantes estaban en estadios avanzados de demencia (superiores al estadio 4 de la escala GDS).

\section{RESULTADOS}

En una primera búsqueda se obtuvieron 1.689 estudios, descartándose 1.676 por no cumplir con los criterios de inclusión, estar duplicados, o no ser el objeto del estudio la Reminiscencia, revisándose finalmente 13 estudios. En la siguiente tabla (Tabla 1) se muestran los artículos seleccionados y sus características principales.

Tabla 1. Características de los Estudios Revisados.

\begin{tabular}{|c|c|c|c|c|}
\hline Autores & $\begin{array}{l}\text { Características del Estudio y } \\
\text { Sesiones Empleadas }\end{array}$ & $\begin{array}{c}\text { Participantes } \\
\text { (n) }\end{array}$ & $\begin{array}{l}\text { Variables } \\
\text { Evaluadas }\end{array}$ & Resultados \\
\hline \multirow{3}{*}{$\begin{array}{l}\text { Allen, AP, } \\
\text { Doyle, C.,'y } \\
\text { Roche, RA } \\
(2020)\end{array}$} & Comunidad & & & \\
\hline & $\begin{array}{l}\text { Personas mayores sin deterioro cognitivo } \\
\text { Grupos de } 3-7 \text { participantes } \\
6 \text { sesiones/semana } \\
60 \text { minutos/sesión }\end{array}$ & \multirow{2}{*}{$\begin{array}{c}\text { N:36 } \\
\text { GE: } 14 \\
\text { GC (activo): } 11 \\
\text { GC (lista de } \\
\text { espera): } 11\end{array}$} & \multirow{2}{*}{$\begin{array}{l}\text { Calidad de } \\
\text { vida } \\
\text { Satisfacción } \\
\text { con la vida }\end{array}$} & \multirow{2}{*}{$\begin{array}{l}\text { GE: No diferencias } \\
\text { significativas respecto } \\
\text { al GC }\end{array}$} \\
\hline & $\begin{array}{l}\text { GE: Programa de Reminiscencia semiestructurado } \\
\text { GC (activo): Sin intervención en Reminiscencia } \\
\text { GC: Lista de espera }\end{array}$ & & & \\
\hline
\end{tabular}


Grupos de 6 a 10 personas

Ching, Y., 1 ó 2 sesiones/semana

Ya, Y., Chia, 8 semanas

L., y Hsiu, L. 60 minutos/sesión (2020)

GE: Intervención Reminiscencia grupal
GC: No intervención

Centro residencial

Grupos de 6 personas

1 sesión/semana
8 semanas

Lök et ál. 60 minutos/sesión

(2019)

El GE y GC, se dividió en 5 grupos cada uno

GE: Intervención Reminiscencia grupal

GC: No intervención
$\mathrm{N}: 24$

GE: 12

GC: 12
Sentido de vida
GE: Mejoras significativas en sentido de vida respecto al GC

Centro residencial

Grupos de 5 a 10 personas

Una sesión/semana

Siverová, J., 8 semanas

Centro residencial
Grupos de 5 a 10 personas
Una sesión/semana

y Bužgová, R. R0-60 minutos/sesión

(2018)

GE: Las intervenciones involucran TR con un enfoque narrativo

GC: Atención habitual

\section{Comunidad}

Aşiret, GD, 1 visita domiciliaria/semana
y Dutkun, M 8 semanas
30-45 minutos

Sesiones individuales en Terapia de Reminiscencia (TR)

\section{$\mathrm{N}: 50$}

GE: 27

GC: 23

N: 116

GE: 59

GE:30

GC:30 Calidad de
vida
GE: Mejores

puntuaciones en

calidad de vida tras

intervención

Comparación GE/GC:

Mejores puntuaciones en GE que en GC

(

Satorres, E, Comunidad

Viguer, $P$,

Viguer, $\mathrm{P}_{\text {, }}$, Sin deterioro cognitivo ni depresión
Fortuna, $\mathrm{FB}$,

y Meléndez, 10 sesiones Reminiscencia

JC (2018) 2hora/sesión
$N: 150$

GE:77

GC: 73

GE: Mejoría en

calidad de vida (principalmente las $\begin{array}{cl}\text { Calidad de } & \text { áreas de salud mental } \\ \text { vida } & \text { y participación social). }\end{array}$

Afrontamiento Mejores puntuaciones en actitudes hacia el envejecimiento y la vejez.

GE: Mejores puntuaciones en adaptación que el GC

La TR aumentó la adaptación de mujeres mayores a la vejez

GE: Diferencias significativas en la resolución de problemas, reevaluación positiva y búsqueda de apoyo social respecto al GC

Los efectos disminuyeron después de 3 meses, pero se encontraron algunas diferencias en el GE obteniendo puntuaciones más altas en la resolución de problemas y reevaluación positiva y menor expresión emocional.

GE: Mejores puntuaciones en

\section{Comunidad}

Sin deterioro cognitivo

Viguer, $P_{\text {, }} 10$ sesiones

Satorres, 'E, 10 sesiones

Fortuna, FB, 2 horas

y Meléndez,

JC (2017)

GE: Intervención en Reminiscencia

GC: Lista de espera (recibirán la intervención unos meses después. Evaluaciones a la vez)
$\mathrm{N}: 160$

GE: 84

GC: 84

Satisfacción con la vida

Bienestar psicológico satisfacción con la

vida y bienestar

psicológico.

Los efectos permanecieron después de tres meses en el caso de la satisfacción de la vida y algunas dimensiones del bienestar psicológico. 


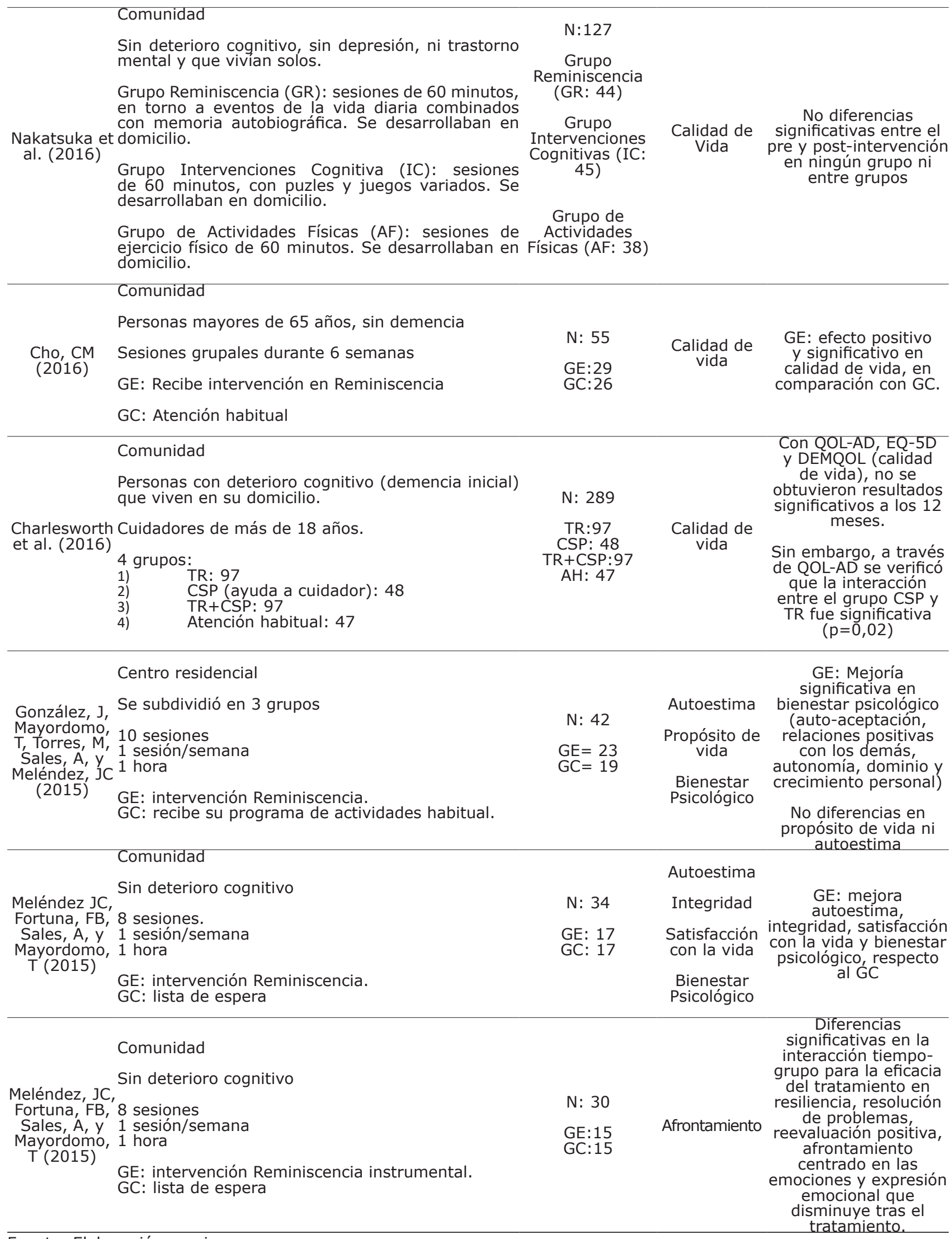

Fuente: Elaboración propia. 
Teniendo en cuenta los estudios en Reminiscencia seleccionados, el 69,23\% se realizan en comunidad y el $30,77 \%$ en centro residencial. El $84,61 \%$ tienen grupo experimental y control; por el contrario, el $15,38 \%$ son estudios de intervención individual o que se comparan entre ellos sin disponer de grupo control.

En cuanto a las variables adaptativas, el 46,15\% estudia la calidad de vida, el 23,07\% satisfacción con la vida y bienestar psicológico, el 15,38 \% autoestima, afrontamiento y adaptación; el 7,69\% sentido de vida, propósito de vida e integridad.

Respecto a los resultados obtenidos tras la realización de una intervención en Reminiscencia, la totalidad de los estudios analizados presentan diferencias significativas en el GE frente al GC en sentido de vida, propósito de vida, bienestar psicológico, integridad, afrontamiento y adaptación, 2 de cada $3(66,66 \%)$ en satisfacción con la vida, y la mitad de los estudios $(50 \%)$ en calidad de vida y autoestima.

En relación con las características de la terapia, el número de sesiones es de 8-10; el 15,38 \% realizan 6 sesiones de intervención; el 46,15\%, 8 sesiones; y el 23,07\%, 10 sesiones, no indicando el número de sesiones el $14,40 \%$ de los estudios.

Teniendo en cuenta la frecuencia de las sesiones, se realiza habitualmente una sesión semanal; el 53,84\% realizan una sesión semanal; el 7,69 \%, entre una y dos sesiones semanales; el $7,69 \%$, seis sesiones por semana; y el $30,76 \%$ de los estudios no indican el número de sesiones semanales.

Respecto a la duración de las sesiones, generalmente es de 60 minutos; el 7,69\% tienen una duración 30-45 minutos; el 46,15\%, de 60 minutos; el 15,38\%, de 120 minutos; y el 23,07\% de los estudios no especifican el tiempo de duración de las sesiones.

Para finalizar, y valorando el tamaño del grupo, en centros residenciales es de 10 personas; el $20 \%$, entre 5-7 participantes; el $40 \%$, entre $6-10$; el $20 \%$, entre $5-10$ personas; y el $20 \%, 8$ personas. Dos de los estudios analizados en centros residenciales, no aportan información al respecto.

\section{DISCUSIÓN}

Teniendo en cuenta el análisis de las investigaciones que estudian variables adaptativas con intervención en Reminiscencia en el periodo analizado (2015-2020), observamos que la mayor parte de las investigaciones se realizan en comunidad (Allen, Doyle y Roche, 2020; Aşiret y Dutkun, 2018; Satorres, Viguer, Fortuna y Meléndez, 2018; Nakatsuka et al., 2016; Cho, 2016; Charlesworth et al., 2016; Meléndez, Fortuna, Sales y Mayordomo, 2015).

Las intervenciones en centros residenciales (Ching, Ya, Chia y Hsiu, 2020; Lök et ál., 2.019; Siverová y Bužgová, 2018; González, Mayordomo, Torres, Sales y Meléndez, 2015), cuentan con un programa grupal de 8-10 sesiones, una sesión por semana con una duración de 60 minutos, 10 participantes, con grupo experimental y grupo control. Las variables adaptativas más estudiadas son calidad de vida (Allen, Doyle y Roche, 2020; Lök et ál., 2019; Siverová y Bužgová, 2018; Nakatsuka etal., 2016; Cho, 2016 y Charlesworth et al., 2016); satisfacción con la vida y bienestar psicológico (Viguer, Satorres, Fortuna y Meléndez, 2017; Meléndez, Fortuna, Sales y Mayordomo, 2015b), presentando resultados positivos tras la intervención en sentido de vida (Ching, Ya, Chia y Hsiu, 2020); propósito de vida (González, Mayordomo, Torres, Sales y Meléndez, 2015); bienestar psicológico e integridad (Meléndez, Fortuna, Sales y Mayordomo, 2015b); afrontamiento (Siverová y Bužgová, 2018); adaptación (Aşiret, y Dutkun, 2018; Satorres, 
Viguer, Fortuna y Meléndez, 2018) y satisfacción con la vida (Allen, Doyle y Roche, 2020; Viguer, Satorres, Fortuna, y Meléndez, 2017).

La presencia de síntomas depresivos se asocia con el uso desadaptativo de las funciones de la Reminiscencia, centrándose en la memoria de hechos pasados para escapar de los desafíos del presente. Al centrarse en los recuerdos de metas no alcanzadas, la persona se arrepiente del pasado y se concentra en la pérdida de significado; el uso reducido de estrategias de afrontamiento basadas en el análisis lógico de los problemas, junto con una personalidad emocionalmente inestable y tendencia a las emociones negativas, se asocian con una mayor probabilidad de síntomas depresivos y, por lo tanto, peor salud mental (Fernández, Ros, Escribano y Serrano, 2020).

Las funciones de la Reminiscencia son relevantes en la vejez y, por ello, autores como Hofer, Busch, Au, Poláčková, Tavel, y Tsien (2020), afirman que la función de enseñar/informar y preparar para la muerte se asocian de manera positiva con el comportamiento generativo facilitando el bienestar que, a su vez, está relacionado con el significado y sentido de vida. Pensar en la muerte puede motivar a las conductas generativas, pues el comportamiento generativo ofrece la oportunidad de dejar un legado que trasciende.

Según Dezutter, Toussaint y Dewitte (2020), las personas que superan exitosamente el estadio de integración eriksoniano muestran pocos síntomas depresivos y se sienten más satisfechas con la vida y con su estado de salud. Por contra, las personas que no superan exitosamente el estadio de integración eriksoniano, permanecen en un estado de desesperación, tienen puntuaciones altas en sintomatología depresiva, bajas en satisfacción con la vida y están en riesgo de tener problemas de salud mental. La integración de las experiencias de vida en un todo se asocia con el envejecimiento exitoso (Ching, Ya, Chia, y Hsiu, 2020).

La Terapia de Reminiscencia conecta las experiencias pasadas de las personas mayores, permitiéndoles relatar y compartir sus historias de vida, obtener apoyo emocional y aliviar las emociones negativas, la depresión y la soledad (Lök et ál., 2019).

Cuando la Terapia de Reminiscencia es grupal y estructurada, ayuda a las personas mayores a mejorar la autoestima, la confianza en sí mismos, el bienestar emocional y la satisfacción al recordar y reconstruir experiencias (Kousha, Sayedi, Moghaddam y Matlabi, 2020), razones por las cuales es un tipo de terapia no farmacológica muy útil en centros residenciales, pues mejora los síntomas depresivos, aumenta la confianza, la satisfacción y el significado de vida (Lök et ál., 2019).

\section{CONCLUSIONES}

Con todo ello, consideramos que este trabajo podría ser relevante para motivar la investigación y la intervención sobre el tema de la Reminiscencia y sus beneficios potenciales al mejorar la ansiedad (Lopes, Afonso y Ribeiro, 2016), síntomas depresivos, habilidades cognitivas, bienestar psicológico, satisfacción vital, calidad de vida y compromiso social (Shropshire, 2020; İnel y Simsek, 2019; Hsieh et ál., 2010), capacidad adaptativa, resiliencia, y significado de vida favoreciendo la integridad (Meléndez, Fortuna, Sales y Mayordomo, 2015a), obteniéndose además beneficios positivos para la salud en personas mayores (İnel y Simsek, 2019; Lök, Bademli, y Selçuk-Tosun, 2019; Shropshire, 2020). 


\section{REFERENCIAS}

Allen, AP, Doyle, C., y Roche, RA. (2020). The Impact of Reminiscence on Autobiographical Memory, Cognition and Psychological Well-Being in Healthy Older Adults. Europe's Journal of Psychology, 16 (2), 317-330. https://doi.org/10.5964/ejop.v16i2.2097

Aşiret, GD y Dutkun, M. (2018). The effect of reminiscence therapy on the adaptation of elderly women to old age: a randomized clinical trial. Complementary therapies in Medicine, 41, 124-129. https://doi.org/10.1016/j.ctim.2018.09.018.

Bohlmeijer, ET, Westerhof, GJ, y Emmerik-de Jong, M. (2008). The effects of integrative reminiscence on meaning in life: results of a quasi-experimental study. Aging and Mental Health, 12 (5), 639-646. https://doi.org/10.1080/13607860802343209

Butler, RN. (1963). The life review: an interpretation of reminiscence in the aged. Psychiatry, 26 (1), 65-76. https://doi.org/10.1080/00332747.1963.11023339

Charlesworth, G., Burnell, K., Crellin, N., Hoare, Z., Hoe, J., Knapp, M., y Orrell, M. (2016). Peer support and reminiscence therapy for people with dementia and their family carers: a factorial pragmatic randomised trial. Journal of Neurology, Neurosurgery and Psychiatry, 87 (11), 1218-1228. https://doi.org/10.1136/jnnp-2016-313736

Ching, Y, Ya, Y, Chia, L, y Hsiu, L. (2020). Effect of group reminiscence therapy on depression and perceived meaning of life of veterans diagnosed with dementia at veteran homes. Social Work in Health Care, 59 (2), 75-90. https://doi.org/10.1080/00981389.2019.1710320

Cho, CM. (2016). The effects of reminiscence therapy on cognitive function, depression and quality of life among the community-dwelling elderly, International Journal of Geriatric Psyquiatry, 23 (4), 545-551. https://doi.org/10.1002/gps.4980

Cortés, IM. (2016). Reminiscencias a través del tiempo. Del archivo fotográfico a la gráfica expandida (Doctoral dissertation). Universidad Politécnica de Valencia. http://hdl.handle. net/10251/69746

Dezutter, J., Toussaint, L., y Dewitte, L. (2020). Finding a balance between integrity and despair: a challenging task for older adults in residential care. Journal of Adult Development, 27 (2), 147-156. https://doi.org/10.1007/s10804-019-09332-1

Duru, G., y Kapucu, S. (2016). The effect of reminiscence therapy on cognition, depression, and activities of daily living for patients with Alzheimer disease. Journal of Geriatric Psychiatry and Neurology, 29 (1), 31-37. https://doi.org/10.1177/0891988715598233

Erikson, EH. (1982). Identity and the life cycle. WW. Norton y Company. https://www.google. com/search?sxsrf=ALeKk00IZGbqx6eRRa9WqjKCcmnv5cwZRw\%3A1607432978207\&ei=E nvPX6aUDKLkgwebnJZg\&q=Erikson\%2C+E+\%281982\%29.+Identity+and+the+life+cycle .+Ciudad\%3A+WW. +Norton+y+Company.\&oq=Erikson\%2C+EH+\%281982\%29. +Identity +and+the+life+cycle.+Ciudad\%3A+WW.+Norton+y+Company.\&gs Icp=CgZwc3ktYWIQDF AAWABg81xoAHAAeACAAQCIAQCSAQCYAQCqAQdnd3Mtd216\&sclient=psy-ab\&ved=0ahUKE wim5am9ur7tAhUi8uAKHRuOBQwQ4dUDCA0 
Martínez-Callado et al - Reminiscencia y Variables Adaptativas en Personas Mayores

Fernández, D., Ros, L., Escribano, F., y Serrano, JP. (2020). Reminiscence, personality, coping and mood state in institutionalised older adults: a cross-sectional study. Psychogeriatrics, 20 (3), 310-3. https://doi.org/10.1111/psyg. 12500

González, J., Mayordomo, T., Torres, M., Sales, A., y Meléndez, JC. (2015). Reminiscence and dementia: a therapeutic intervention. International Psychogeriatrics, 27 (10), 1731-1737. https://doi.org/10.1017/S1041610215000344

Hofer, J, Busch, H, Au, A, Poláčková, I, Tavel, P, y Tsien, T. (2020). Reminiscing to teach others and prepare for death is associated with meaning in life through generative behavior in elderlies from four cultures. Aging and Mental Health, 24 (5), 811-819. https://doi.org/10.1 $\underline{080 / 13607863.2018 .154856}$

Hsieh, CJ, Chang, C, Su, SF, Hsiao, YL, Shih, YW, Han, WH, y Lin, CC. (2010). Reminiscence group therapy on depression and apathy in nursing home residents with mild-to-moderate dementia. Journal of Experimental and Clinical Medicine,2 (2), 72-78. https://doi. org/10.1186/1471-244X-12-189

İnel, A., y Simsek, N. (2019). The effect of reminiscence therapy with internet-based videos on cognitive status and apathy of older people with mild dementia. Journal of Geriatric Psychiatry and Neurology, 32 (2), 104-113. https://doi.org/10.1177/0891988718819864

Irazoki, E, García, JA, Sánchez, J, y Franco, M. (2017). Eficacia de la Terapia de Reminiscencia grupal en personas con demencia. Revisión sistemática y metaanálisis. Rev Neurol, 65 (10), 447-456.

Kousha, A, Sayedi, A, Moghaddam, HR, y Matlabi, H. (2020). El Protocolo iraní de Reminiscencia del grupo y calidad de vida relacionada con la salud entre las personas mayores institucionalizadas. Revista de Atención Sanitaria Multidisciplinaria, 13, 1027.

Lök, N., Bademli, K., y Selçuk, A. (2019). The effect of reminiscence therapy on cognitive functions, depression, and quality of life in Alzheimer patients: randomized controlled trial. International Journal of Geriatric Psychiatry, 34 (1), 47-53. https://doi.org/10.1002/gps.4980

Lopes, TS, Afonso, RM, y Ribeiro, OM. (2016). A quasi-experimental study of a reminiscence program focused on autobiographical memory in institutionalized older adults with cognitive impairment. Archives of Gerontology and Geriatrics, 66, 183-192. https://doi.org/10.1016/j. archger.2016.05.007

Meléndez, JC, Fortuna, FB, Sales, A, y Mayordomo, T. (2015a). The effects of instrumental reminiscence on resilience and coping in elderly. Archives of Gerontology and Geriatrics, 60 (2), 294-298. https://doi.org/10.1080/17439760.2014.936968

Meléndez, JC, Fortuna, FB, Sales, A, y Mayordomo, T. (2015b). Effect of integrative reminiscence therapy on depression, well-being, integrity, self-esteem, and life satisfaction in older adults. The Journal of Positive Psychology, 10 (3), 240-247. https://doi.org/10.1080/17439760.20 14.936968

Nakatsuka, M, Nakamura, K, Kasai, M, Nakai, M y Meguro, K. (2016). The group reminiscence approach can increase self-awareness of memory deficits and evoke a life review in people with mild cognitive impairment: the Kurihara Project Data. Journal of the American Medical Directors Association, 17 (6), 501-507. https://doi.org/10.1016/j.jamda.2015.11.009 
Satorres, E, Viguer, P, Fortuna, FB, y Meléndez, JC. (2018). Effectiveness of instrumental reminiscence intervention on improving coping in healthy older adults. Stress and Health, 34 (2), 227-234. https://doi.org/10.1002/smi.2776

Shropshire, M. (2020). Reminiscence intervention for community-dwelling older adults without dementia: a literature review. British Journal of Community Nursing, 25 (1), 40-44. https:// doi.org/10.12968/bjen.2020.25.1.40

Siverová, J y Bužgová, R. (2018). The effect of reminiscence therapy on quality of life, attitudes to ageing, and depressive symptoms in institutionalized elderly adults with cognitive impairment: a quasi-experimental study. International Journal of Mental Health Nursing, 27 (5), 1430-1439. https://doi.org/10.1111/inm.12442

Van Bogaert, P, Tolson, D, Eerlingen, R, Carvers, D, Wouters, K, Paque, K, Engelborghs, S. (2016). SolCos model-based individual reminiscence for older adults with mild to moderate dementia in nursing homes: a randomized controlled intervention study. Journal of Psychiatric and Mental Health Nursing, 23 (9-10), 568-575. https://doi.org/10.1111/jpm.12336

Viguer, P, Satorres, E, Fortuna, FB, y Meléndez, JC. (2017). A follow-up study of a reminiscence intervention and its effects on depressed mood, life satisfaction, and well-being in the elderly. The Journal of Psychology, 151 (8), 789-803. https://doi.org/10.1080/00223980.2017.13 $\underline{93379}$

Webster, JD. (1997). The reminiscence functions scale: a replication. International Journal of Aging and Human Development, 44 (2), 137-148.

Westerhof, GJ, Bohlmeijer, E y Webster, JD. (2010). Reminiscence and mental health: a review of recent progress in theory, research and interventions. Ageing and Society, 30 (4), 697-721. 